\title{
Flexible Tethered Kite with Moveable Attachment Points, Part II: State and Wind Estimation
}

\author{
Paul Williams, ${ }^{*}$ Bas Lansdorp, Wubbo Ockels \\ Delft University, The Netherlands
}

\begin{abstract}
Tethered kite technology is one potential means of harnessing energy available in high altitude winds. In an efficient and practical system, the kite is required to fly in cyclic patterns to maximize net power produced per cycle. At the same time, the tether length must be controlled to ensure the system does not expend more energy than it produces. This can be a challenging problem when the intermittency of the wind speed and direction, as well as unsteady wind components, are taken into account. This paper is the second in a series of two that studies the dynamics and control of a flexible kite. In this part, the dynamic model and trajectories derived in Part I are used to develop a nonlinear dynamic observer. Full state estimation is required to implement one of the proposed feedback tracking controllers. The system dynamics are extremely complex and therefore a method that does not require the computation of vector field derivatives has been chosen. The square-root unscented Kalman filter is implemented to estimate the kite dynamics as well as the true wind speed at the kite.
\end{abstract}

\section{Introduction}

$\mathbf{T}$ HE development of kite technology is considered to be an important requirement for future wind power systems, such as the Laddermill. ${ }^{1-5}$ The Laddermill is a concept that can enable wind energy to be extracted from higher altitudes than can be reached using conventional systems such as wind turbines. Although there have been many proposals for systems that seek to extract the energy prevalent in the wind at higher altitudes, ${ }^{6-28}$ the Laddermill is a concept that has seen much progress in recent years. The advantage of the Laddermill over many other schemes is that the power generation equipment is located on the ground rather than at higher altitudes. This saves a tremendous amount of effort in placing the system at the desired altitude, and also has obvious safety implications. Instead, the system uses a series of light-weight kites attached to a variable length tether. The kites are controlled to produce alternating periods of high and low tension in the tether. This can be achieved by flying the kites in the cross-wind direction and changing the kite angle of attack. ${ }^{29}$ A review of high altitude wind power concepts, as well as the development of optimal trajectories for a simplified system are available in Ref. 29. However, in this previous work, the kite dynamics were not explicitly taken into account and it was assumed that the kite angle of attack and roll angle could be controlled. In practice, defining the "kite angle of attack" and "kite roll angle" is nontrivial due to the effects of kite flexibility. This also raises issues related to the implementation of appropriate tracking controllers.

In Part I of this series of papers, a simplified dynamic model representing the kite was derived and trajectory generation and feedback control were applied to establish a control strategy for maneuvering a flexible kite system. The kite is controlled by moving the positions of the tether attachments on the two wing-tips. The mechanism used to achieve this is shown in Fig. 1. The feedback controller in Part I was based on a receding horizon approach, but uses the linearized dynamics rather than the full nonlinear dynamics. The full nonlinear dynamics are used to construct the timevarying reference trajectories. One of the drawbacks with such an approach is that the full state vector is assumed to be available to the feedback controller. For a complex system like

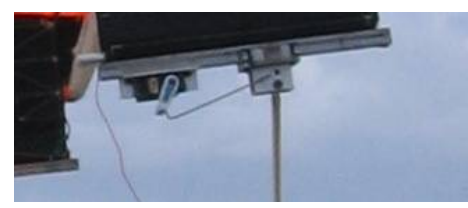

Fig. 1 Sliding mechanism along kite edge.

\footnotetext{
*AIAA Member, tethered.systems@gmail.com.
} 
a kite, this would be an impossible task. Only a limited number of sensors are available, and only the minimum should be used to keep the system weight down. Furthermore, the extreme flexibility of the kite system makes it a challenge from a control perspective.

In this Part, the objective is to design a nonlinear observer and state estimator for the kite model derived in Part I. The control actuators for the kite are the positions of the tether attachment points, which are controlled by a sliding mechanism on the edge of the kite, as shown in Fig. 1. By moving the attachment points, a torque is produced due to the tension in the tether(s), which alters the local angle of attack of the kite. To implement the feedback controller developed in Part I, it is necessary to have knowledge of the kite state vector as well as the mean wind speed. Recent advances in nonlinear estimation theory mean that it is possible to develop sophisticated derivative-free filters for complex systems such as a tethered kite. This paper demonstrates that it is possible to reconstruct the dynamics of a flexible kite using an unscented Kalman filter. The reasons for this choice as well as the issues in implementation of the filter are discussed.

The structure of this paper is as follows: First, the dynamic model of the kite is reviewed; next, options for designing the dynamic estimator are considered and the method employed is reviewed; numerical results illustrating the effectiveness of the filter are given; and finally, some conclusions are drawn.

\section{Simplified Dynamic Model of a Tethered Kite}

The kite model that is used for the dynamic estimation is derived more completely in Part I. However, because the model is essential to discussing the implementation of the filter, a brief overview of the model features is included here. The kite is modeled as consisting of two flat plates articulated by a frictionless hinge. The connecting tethers are modeled as elastic, but straight. A representation of the model is shown in Fig. 2, which defines the coordinate systems used.

The hinge point is used as the reference point for the translational motion of the kite. Its position is defined by the Cartesian coordinates $(x, y, z)$. The hinge point is nominally at $(0,0,0)$ for the equilibrium configuration of the kite. The inertial axes are defined such that the $x$-axis points predominantly in the direction opposite to the wind, the $z$-axis points vertically down, and the $y$-axis completes the right-handed triad. Body frames are also used, which are attached to the centers of mass of each plate, as shown in Fig. 2.

The two tethers that are attached to the kite are joined at a bridle, which is modeled as a lumped mass, denoted $m_{b}$ in Fig. 2. The complete system of tethers are modeled as extensible springs following Hooke's law. The position of the mass $m_{b}$ is denoted by the coordinates $\boldsymbol{R}_{b}=\left(x_{b}, y_{b}, z_{b}\right)$.

The body frame of each plate is related to the inertial frame via the orthogonal transformation

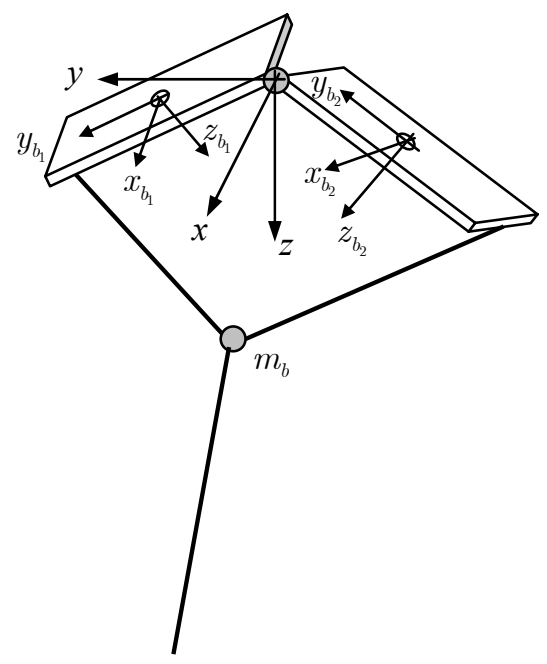

Fig. 2 Simple tethered kite model.

$$
C_{I_{j}}^{B}=\left[\begin{array}{ccc}
\cos \theta_{j} \cos \psi & \cos \theta_{j} \sin \psi & -\sin \theta_{j} \\
-\cos \phi_{j} \sin \psi+\sin \phi_{j} \sin \theta_{j} \cos \psi & \cos \phi_{j} \cos \psi+\sin \phi_{j} \sin \theta_{j} \sin \psi & \sin \phi_{j} \cos \theta_{j} \\
\sin \phi_{j} \sin \psi+\cos \phi_{j} \sin \theta_{j} \cos \psi & -\sin \phi_{j} \cos \psi+\cos \phi_{j} \sin \theta_{j} \sin \psi & \cos \phi_{j} \cos \theta_{j}
\end{array}\right]
$$

where $\theta_{j}$ is the pitch of the $j$ th plate, $\phi_{j}$ is the roll of the $j$ th plate, and $\psi$ is the yaw. The yaw angles of both plates are constrained to be the same in the model. The velocity of a point on plate $j$ is given by 


$$
\begin{aligned}
& \dot{\boldsymbol{R}}_{j}^{c m}=\left[\dot{x}-\left(\dot{\theta}_{j} \sin \theta_{j} \cos \psi+\dot{\psi} \cos \theta_{j} \sin \psi\right) X+\left\{\left(\sin \phi_{j} \sin \psi+\cos \phi_{j} \sin \theta_{j} \cos \psi\right) \dot{\phi}_{j}-\left(\cos \phi_{j} \cos \psi+\sin \phi_{j} \sin \theta_{j} \sin \psi\right) \dot{\psi}+\dot{\theta}_{j} \sin \phi_{j} \cos \theta_{j} \cos \psi\right\} Y\right. \\
& \dot{y}-\left(\dot{\theta}_{j} \sin \theta_{j} \sin \psi-\dot{\psi} \cos \theta_{j} \cos \psi\right) X+\left\{\left(-\sin \phi_{j} \cos \psi+\cos \phi_{j} \sin \theta_{j} \sin \psi\right) \dot{\phi}_{j}-\left(\cos \phi_{j} \sin \psi-\sin \phi_{j} \sin \theta_{j} \cos \psi\right) \dot{\psi}+\dot{\theta}_{j} \sin \phi_{j} \cos \theta_{j} \sin \psi\right\} Y \\
& \left.\dot{z}-\dot{\theta}_{j} X \cos \theta_{j}+\left(\dot{\phi}_{j} \cos \phi_{j} \cos \theta_{j}-\dot{\theta}_{j} \sin \phi_{j} \sin \theta_{j}\right) Y\right]
\end{aligned}
$$

where $X$ and $Y$ are the components of the vector to the center of mass, attachment, or aerodynamic center in the body frame.

The aerodynamic forces are computed by using the relative wind velocity at the center of each of the plates. Thus, the lift and drag coefficients are based on the relative wind velocity at a single point on each side of the kite. The total lift and drag forces are applied at the aerodynamic center of the plate.

The lift and drag coefficients of the plates are approximated via

$$
C_{L_{j}}=C_{L_{\alpha}} \alpha_{j}, \quad C_{D_{j}}=C_{D_{0}}+k C_{L_{j}}^{2}, \quad \alpha \leq \alpha_{\max }
$$

The tension forces acting on the plates give rise to both applied forces and moments. The moments are particularly important because control of the system is sought by moving the positions of the tether attachment points on the kite. For simplicity, we have ignored aerodynamic forces on the bridle mass and will only treat tension and gravity forces. Thus,

$$
m_{b} \ddot{\boldsymbol{R}}_{b}=\boldsymbol{T}_{3}-\boldsymbol{T}_{1}-\boldsymbol{T}_{2}+m_{b} g \boldsymbol{k}
$$

The directions of the tension force vectors are defined in Fig. 3. The tensions acting on the attachment points of the kite can be defined by

$$
\boldsymbol{T}_{j}=\frac{E A}{L_{s_{j}}}\left(\left|\boldsymbol{R}_{b}-\boldsymbol{R}_{j}^{a}\right|-L_{s_{j}}\right) \frac{\boldsymbol{R}_{b}-\boldsymbol{R}_{j}^{a}}{\left|\boldsymbol{R}_{b}-\boldsymbol{R}_{j}^{a}\right|}, j=1,2
$$

where $E A$ is the longitudinal stiffness of the tether, and $L_{s_{j}}$ is the unstrained length of the $j$ th tether.

The tension in the main tether line is defined by

$$
\boldsymbol{T}_{3}=\frac{E A}{L_{s_{3}}}\left(\left|\boldsymbol{R}_{g}-\boldsymbol{R}_{b}\right|-L_{s_{3}}\right) \frac{\boldsymbol{R}_{g}-\boldsymbol{R}_{b}}{\left|\boldsymbol{R}_{g}-\boldsymbol{R}_{b}\right|}
$$

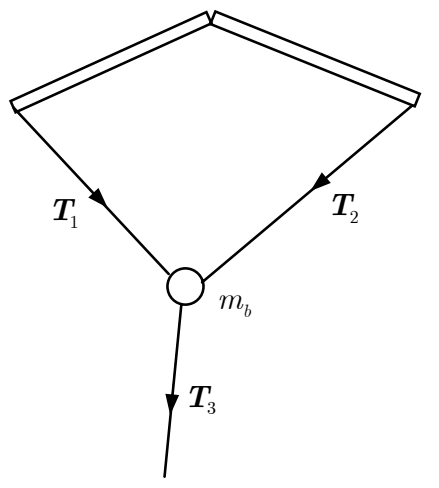

Fig. 3 Tension vector definition.

Further details on the derivation of the full equations of motion are given in Ref. 30. Several important points about the final equations of motion need to be made. First, the equations are inertially coupled and are therefore highly complex when decoupled. Second, the equations of motion are highly nonlinear. Therefore, we are limited in the kinds of filters that can be applied to the system. This issue is discussed in the following section.

\section{Nonlinear Dynamic State and Wind Estimation}

In the results presented in Ref. 30 for time-varying reference trajectories, we have assumed that full state feedback is available for the kite. However, for equilibrium control, it has been demonstrated that a simple PID 
controller is sufficient for stabilizing the kite motion in the presence of unsteady winds. The PID controller does not require detailed knowledge about the full kite system vector, whereas the receding horizon controller assumes a full state vector in the feedback loop. In the mathematical model, this is easily incorporated to test the validity of the controller. However, because the real kite is not merely two plates, for a real system an estimator is required to take the available measurements and output the state vector that corresponds to the reference model. However, in this work, we are using the two plate model as the truth, and hence the estimation problem is somewhat simpler. The goal is to estimate the full kite state vector from a limited number of measurements.

A variety of approaches are available for state estimation, including the Kalman filter ${ }^{31}$ and particle filters. ${ }^{32}$ The Kalman filter is able to perform state estimation in an optimal manner for linear systems in which the probabilistic distributions for the variables are Gaussian. For nonlinear systems, the extended Kalman filter (EKF) has been the method of choice for several decades. In the EKF, the estimation is no longer optimal because the state distribution is propagated through the model using only a first-order linearization. Thus, the EKF has three major drawbacks: 1) the linear approximations can lead to large errors in the posterior mean and covariance, 2) the filter can often diverge because it is based on local linearization of the system dynamics and measurement equations, and 3) full Jacobians of the vector field and measurement equations are required.

Due to the complexity of the kite system, it is very difficult to derive the required Jacobians that are needed for the EKF. Although the Jacobians could themselves be estimated using finite differences, the problem of filter divergence still remains. As a result of these problems, Julier, Uhlmann and Durrant-Whyte ${ }^{33}$ developed an approach that is able to capture the posterior mean and covariance when propagated through any nonlinear function to second order. The approach relies on the unscented transformation, which is a method for determining the statistics of a random variable after it has undergone a nonlinear transformation. The unscented transformation requires the selection of $2 L+1$ sigma points, where $L$ is the dimension of the state vector. The sigma points capture the mean and covariance of a Gaussian random variable completely. The sigma points are then propagated through the true nonlinear dynamics of the system. In this way, the system Jacobians are not required at all for implementation. The implementation of the algorithm for nonlinear filtering is called the unscented Kalman filter.

The unscented filter has been selected for estimating the kite state vector due to the complexity of the kite equations of motion. Simulations of the kite system are carried out by numerically solving for the second derivatives of the kite generalized coordinates. This must either be achieved via a matrix inversion, or by Gaussian elimination. In any case, this means that the equations of motion are not available analytically in state vector form. One of the key requirements of the EKF is that the Jacobian of the state transition function is available. If the Jacobian is not known analytically, it must be calculated via finite differences, which results in a numerically expensive filter, which is known a priori to be non-optimal. The unscented filter propagates the sigma points directly through the nonlinear model, and hence, the model does not need to be expressible in analytic form. This makes the unscented filter very appealing as a nonlinear observer for the kite system.

One of the problems inherent in all standard implementations of the Kalman filter is that the covariance matrix can become non-positive definite. This can lead to divergence of the filter, even in the case of the unscented filter. To overcome this, the covariance can be updated in square-root form. This helps to prevent round-off errors from propagating through the model and creating ill-conditioned covariance matrices. The particular implementation that is used in this work was presented by van der Merwe and Wan. ${ }^{35}$

\section{A. Square-Root Unscented Kalman Filter}

Most filters are derived for discrete-time nonlinear equations. Any set of differential equations can be converted into discrete-time by means of an explicit propagator, such as an Euler or Runge-Kutta algorithm. In this paper, a fourth order Runge-Kutta algorithm is used in the state estimation to improve stability. However, this comes with a four-fold increase in computational demands. The system model is assumed to be of the form

$$
\begin{gathered}
\boldsymbol{x}_{k+1}^{s}=\boldsymbol{f}\left(\boldsymbol{x}_{k}^{s}, \boldsymbol{w}_{k}, \boldsymbol{u}_{k}, t_{k}\right) \\
\boldsymbol{y}_{k}=\boldsymbol{h}\left(\boldsymbol{x}_{k}^{s}, \boldsymbol{v}_{k}, \boldsymbol{u}_{k}, t_{k}\right)
\end{gathered}
$$

where $\boldsymbol{x}_{k}^{s}$ is the state vector, $\boldsymbol{w}_{k}$ is a zero mean Gaussian process noise with covariance $\boldsymbol{Q}_{k}, \boldsymbol{u}_{k}$ is the control input, $\boldsymbol{v}_{k}$ is a zero mean Gaussian measurement noise with covariance $\boldsymbol{R}_{k}$, and $\boldsymbol{y}_{k}$ are the measured outputs. The functions $\boldsymbol{f}()$ and $\boldsymbol{h}()$ are general nonlinear functions, but are assumed to be known.

In the following, we implicitly augment the state vector with the process and measurement noise as follows 


$$
\boldsymbol{x}_{k}=\left[\begin{array}{c}
\boldsymbol{x}_{k}^{s} \\
\boldsymbol{w}_{k} \\
\boldsymbol{v}_{k}
\end{array}\right]
$$

The first step in the filter is to compute the set of sigma points as follows

$$
\mathcal{X}_{k-1}=\left[\hat{\boldsymbol{x}}_{k-1}, \hat{\boldsymbol{x}}_{k-1}+\gamma \boldsymbol{S}_{k}, \hat{\boldsymbol{x}}_{k-1}-\gamma \boldsymbol{S}_{k}\right]
$$

where $\hat{\boldsymbol{x}}$ is the mean estimate of the state vector, $\boldsymbol{S}_{k}$ is the Cholesky form of the covariance matrix, and the parameter $\gamma$ is defined by

$$
\gamma=\sqrt{L+\lambda}
$$

and $\lambda=\alpha^{2}(L+\kappa)-L$ is a scaling parameter, with the values of $\alpha$ and $\kappa$ selected appropriately []. The sigma points are then propagated through the nonlinear dynamics as follows

$$
\mathcal{X}_{k \mid k-1}^{*}=\boldsymbol{f}\left(\mathcal{X}_{k-1}, \boldsymbol{u}_{k}, t_{k}\right)
$$

The predicted mean for the state estimate is calculated from

$$
\hat{\boldsymbol{x}}_{k}^{-}=\sum_{i=0}^{2 L} W_{i}^{\text {mean }} \mathcal{X}_{i, k \mid k-1}^{*}
$$

where

$$
W_{i}^{\text {mean }}= \begin{cases}\frac{\lambda}{L+\lambda}, & i=0 \\ \frac{1}{2(L+\lambda)}, & i=1, \ldots, 2 L\end{cases}
$$

The Cholesky form of the covariance is predicted using

$$
\begin{aligned}
\boldsymbol{S}_{k}^{-} & =\operatorname{qr}\left[\sqrt{W_{1}^{\operatorname{cov}}}\left(\mathcal{X}_{1: 2 L, k \mid k-1}^{*}-\hat{\boldsymbol{x}}_{k}^{-}\right), \sqrt{\boldsymbol{Q}_{k}}\right] \\
\boldsymbol{S}_{k}^{-} & =\text {cholupdate }\left\{\boldsymbol{S}_{k}^{-}, \mathcal{X}_{0, k}^{*}-\hat{\boldsymbol{x}}_{k}^{-}, W_{0}^{\text {cov }}\right\}
\end{aligned}
$$

where

$$
W_{i}^{\mathrm{cov}}=\left\{\begin{array}{cl}
\frac{\lambda}{L+\lambda}+\left(1-\alpha^{2}+\beta\right), & i=0 \\
\frac{1}{2(L+\lambda)}, & i=1, \ldots, 2 L
\end{array}\right.
$$

$\mathrm{qr}\{\}$ represents the QR decomposition of the matrix, and cholupdate \{\} represents the Cholesky factor update. Next, the propagated sigma points are augmented by

$$
\mathcal{X}_{k \mid k-1}=\left[\mathcal{X}_{k \mid k-1}^{*}, \mathcal{X}_{k \mid k-1}^{*}+\gamma \sqrt{\boldsymbol{Q}_{k}}, \mathcal{X}_{k \mid k-1}^{*}-\gamma \sqrt{\boldsymbol{Q}_{k}}\right]
$$

The augmented sigma points are propagated through the measurement equations

$$
\mathcal{Y}_{k \mid k-1}=\boldsymbol{h}\left(\mathcal{X}_{k \mid k-1}, \boldsymbol{u}_{k}, t_{k}\right)
$$

The mean observation is obtained by

$$
\hat{\boldsymbol{y}}_{k}^{-}=\sum_{i=0}^{2 L} W_{i}^{\text {mean }} \mathcal{Y}_{i, k \mid k-1}
$$

The output Cholesky covariance is calculated using

$$
\begin{gathered}
\boldsymbol{S}_{\tilde{\boldsymbol{y}}_{k}}=\operatorname{qr}\left[\sqrt{W_{1}^{\operatorname{cov}}}\left(\mathcal{Y}_{1: 2 L, k}-\hat{\boldsymbol{y}}_{k}^{-}\right), \sqrt{\boldsymbol{R}_{k}}\right] \\
\boldsymbol{S}_{\tilde{\boldsymbol{y}}_{k}}=\text { cholupdate }\left\{\boldsymbol{S}_{\tilde{\boldsymbol{y}}_{k}}, \mathcal{Y}_{0, k}-\hat{\boldsymbol{y}}_{k}^{-}, W_{0}^{\operatorname{cov}}\right\}
\end{gathered}
$$

The cross-correlation matrix is determined from 


$$
\boldsymbol{P}_{\boldsymbol{x}_{k} \boldsymbol{y}_{k}}=\sum_{i=0}^{2 L} W_{i}^{\mathrm{cov}}\left(\mathcal{X}_{i, k \mid k-1}-\hat{\boldsymbol{x}}_{k}^{-}\right)\left(\mathcal{Y}_{i, k \mid k-1}-\hat{\boldsymbol{y}}_{k}^{-}\right)^{T}
$$

The gain for the Kalman update equations is computed from

$$
\mathcal{K}_{k}=\left(\boldsymbol{P}_{\boldsymbol{x}_{k} \boldsymbol{y}_{k}} / \boldsymbol{S}_{\tilde{\boldsymbol{y}}_{k}}^{T}\right) / \boldsymbol{S}_{\tilde{\boldsymbol{y}}_{k}}
$$

The state estimate is updated with a measurement using

$$
\hat{\boldsymbol{x}}_{k}=\hat{\boldsymbol{x}}_{k}^{-}+\mathcal{K}_{k}\left(\boldsymbol{y}_{k}-\hat{\boldsymbol{y}}_{k}^{-}\right)
$$

and the covariance is updated using

$$
\boldsymbol{S}_{k}=\text { cholupdate }\left\{\boldsymbol{S}_{k}^{-}, \mathcal{K}_{k} \boldsymbol{S}_{\tilde{\boldsymbol{y}}_{k}},-1\right\}
$$

The above filter is coded in MATLAB using the same dynamic equations for the kite that were derived in Part I. One of the main differences is that a variable-step integration routine is used to propagate the "true dynamics", whereas a fixed step routine is used in the filtering.

\section{B. Dynamic Estimation of Kite State}

It is desirable to be able to estimate the full state vector of the kite with only a few available measurements. The measurements assumed for the system are the position, velocity, and acceleration of the hinge point, position of the bridle mass, and kite yaw angle. The attachment point positions are also assumed to be available. This implies encoders the feedback the positions of the sliding mechanisms. Other measurements that could be used include the tether tension(s), but these were found to actually degrade the results in some cases. The rationale for the assumed measurements are that an inertial measurement unit could be used to provide the kite measurements, including kite angles. A GPS receiver could also be used to provide the position of the bridle mass. In all cases, the measurement noise covariances are selected as: $0.5 \mathrm{~m}$ for kite positions, $0.6 \mathrm{~m} / \mathrm{s}$ for velocities, and $0.4 \mathrm{~m} / \mathrm{s}^{2}$ for accelerations. The yaw angle measurement covariance is assumed to be $20 \mathrm{deg}$. The bridle mass position error covariance is assumed to be $0.6 \mathrm{~m}$. The kite system parameters are given in Table 1 .

\section{Table 1 Nominal system parameters.}

\begin{tabular}{lc}
\hline \hline \multicolumn{1}{c}{ Parameter } & Value \\
\hline Plate width, $W$ & $2 \mathrm{~m}$ \\
Plate length, $L$ & $10 \mathrm{~m}$ \\
Plate mass, $m$ & $10 \mathrm{~kg}$ \\
Chordwise location of a.c. & $30 \%$ \\
Spanwise location of a.c. & $50 \%$ \\
Air density & $1.225 \mathrm{~kg} / \mathrm{m}^{3}$ \\
$C_{L_{\alpha}}$ & $4.4 / \mathrm{rad}$ \\
$C_{D_{0}}$ & 0.03 \\
$k$ & 0.1 \\
Bridle mass, $m_{b}$ & $15 \mathrm{~kg}$ \\
Length of grounding tether & $300 \mathrm{~m}$ \\
Tether stiffness, $E A$ & $10000 \mathrm{~N}$ \\
Length of bridle tethers & $20 \mathrm{~m}$ \\
\hline \hline
\end{tabular}

Several case studies are presented to assess the effectiveness of estimating the kite attitude, as well as the corresponding state velocities. In the first case, the full dynamics of the kite are desired to be estimated and the wind state is assumed to be known. Two examples are used: 1) Stationkeeping, and 2) time-varying trajectory simulation. For the stationkeeping scenario, an initial random disturbance is given to the kite, followed by prescribed inputs to the attachment point positions at $20 \mathrm{sec}(2 \mathrm{sec}$ duration) and $50 \mathrm{sec}$ (4 sec duration). The inputs to the attachment points cause the kite to move away from equilibrium, after which the controller attempts to correct the kite attitude and position. For the path 1 simulation, the kite is simulated for 3 cycles without any disturbances. 


\section{Stationkeeping State Estimation}

An example of the noisy data used in the state estimation process is given in Fig. 4. As is typically the case with noisy data, the true motion of the kite is very difficult to discern from Fig. 4. The unscented filter is implemented with a sampling rate of $100 \mathrm{~Hz}$, with a process noise covariance of $\boldsymbol{Q}=\left[\left[10^{-}\right.\right.$ $\left.\left.{ }^{6}\right]_{11 \times 1}, 0.4,0.4,0.4,\left[10^{-5}\right]_{5 \times 1}, 0.4,0.4,0.4\right]$, where the state vector is defined as $\boldsymbol{x} \triangleq\left[x, y, z, \psi, \theta_{1}, \phi_{1}, \theta_{2}, \phi_{2}, x_{b}, y_{b}, z_{b}\right.$, $\left.\dot{x}, \dot{y}, \dot{z}, \dot{\psi}, \dot{\theta}_{1}, \dot{\phi}_{1}, \dot{\theta}_{2}, \dot{\phi}_{2}, \dot{x}_{b}, \dot{y}_{b}, \dot{z}_{b}\right]$.

The quality of the state estimation is shown in Fig. 5 , which gives the hinge point and bridle position as a function of time. The truth is shown as the green line and the state estimate is shown as the black line. The initial state estimate is chosen as a random perturbation around the truth. It can be seen that the position

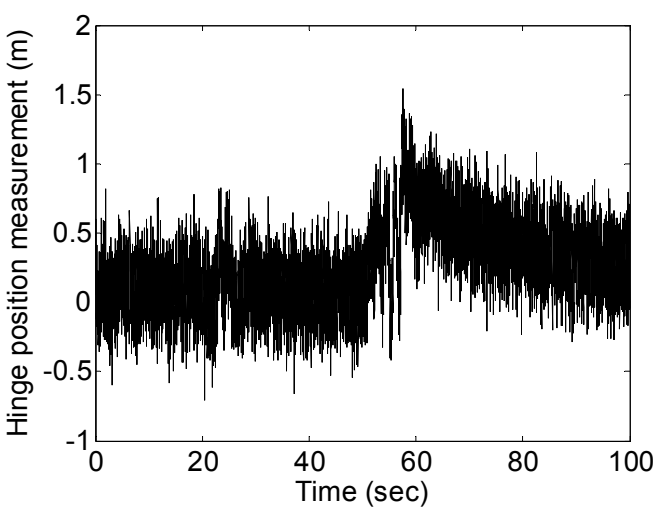

Fig. 4 Example of $z$-hinge position measurements. estimates converge very quickly to the true states. The variation in the states is captured excellently. The $z$-position appears to be slightly more sensitive than the horizontal positions. Fig. 6 shows the kite attitude estimation is performed extremely well. The filter converges to the true trajectory quite quickly. In particular, the yaw angle of the kite is estimated extremely well, despite the fact that yaw is usually hard to estimate for systems moving at low velocities. The kite and bridle velocity estimates are shown in Fig. 7, and Fig. 8 shows the kite attitude rates. The results clearly show that the unscented filter is able to capture the variation in the kite states very well with limited noisy measurements. Furthermore, the results also establish the effectiveness of the closed-loop controller, which stabilizes the kite after the applied disturbances. 

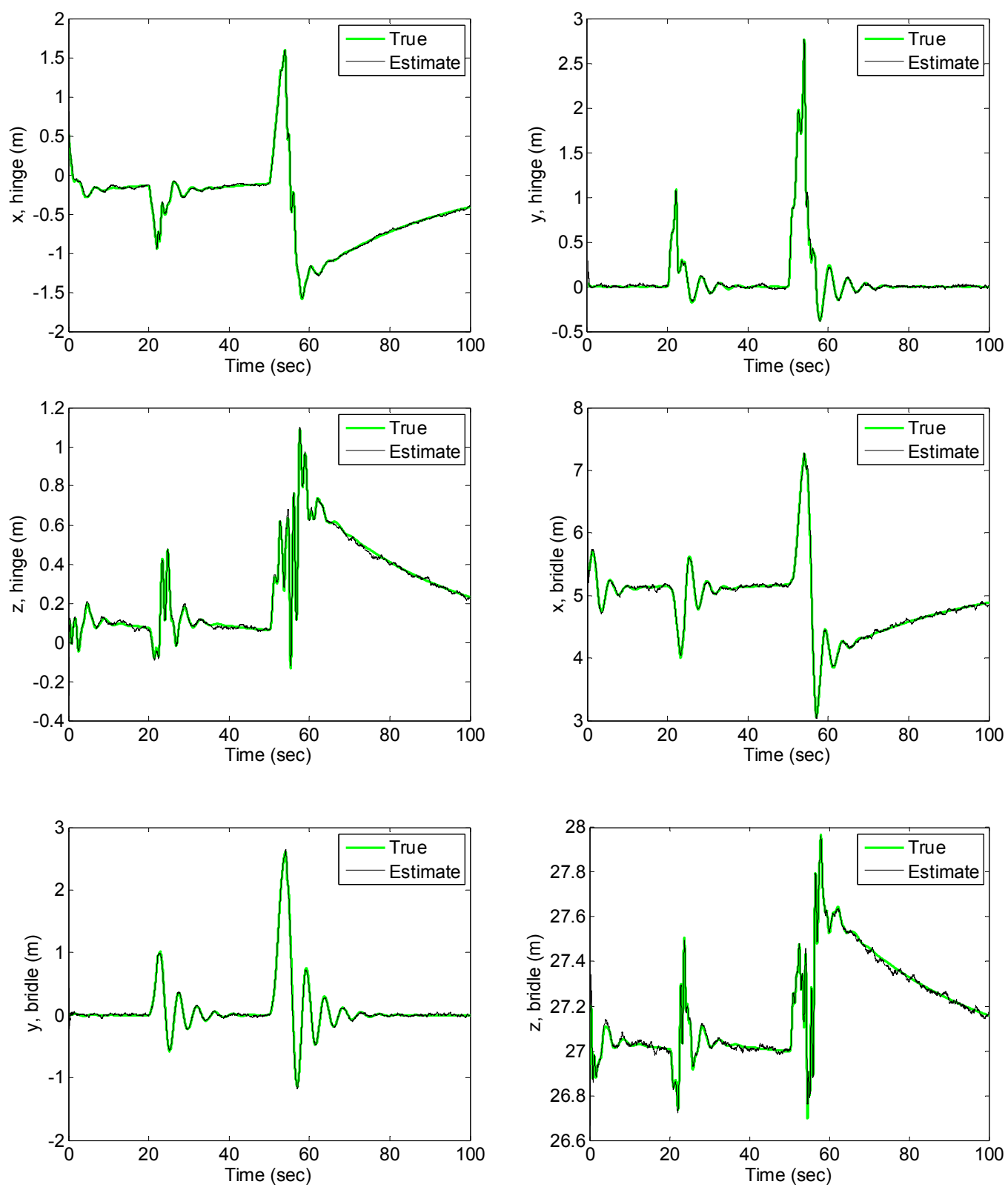

Fig. 5 Kite and bridle position time history due to attachment point disturbance, showing estimated and truth. 

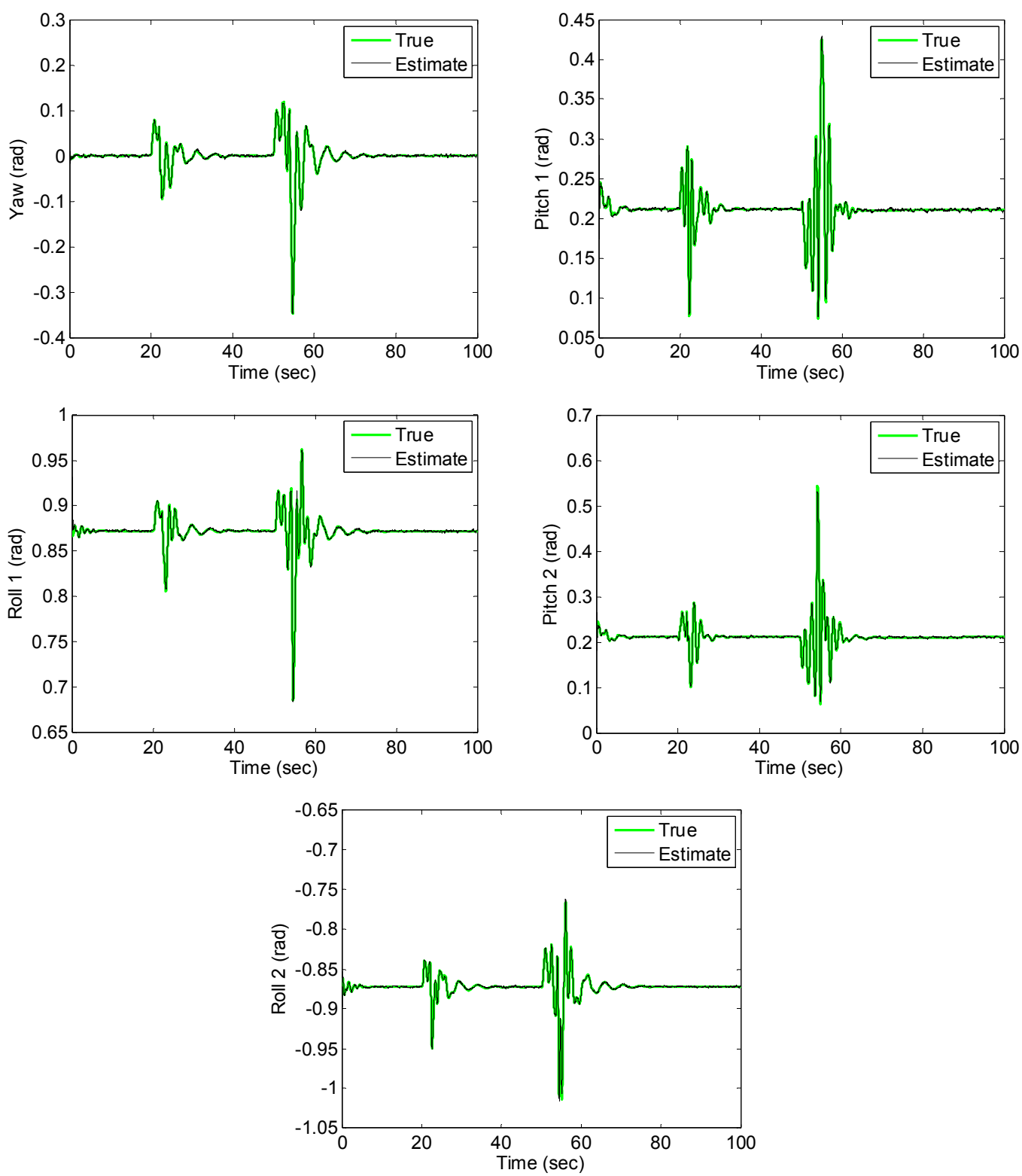

Fig. 6 Kite attitude time history due to attachment point disturbance, showing estimated and truth. 

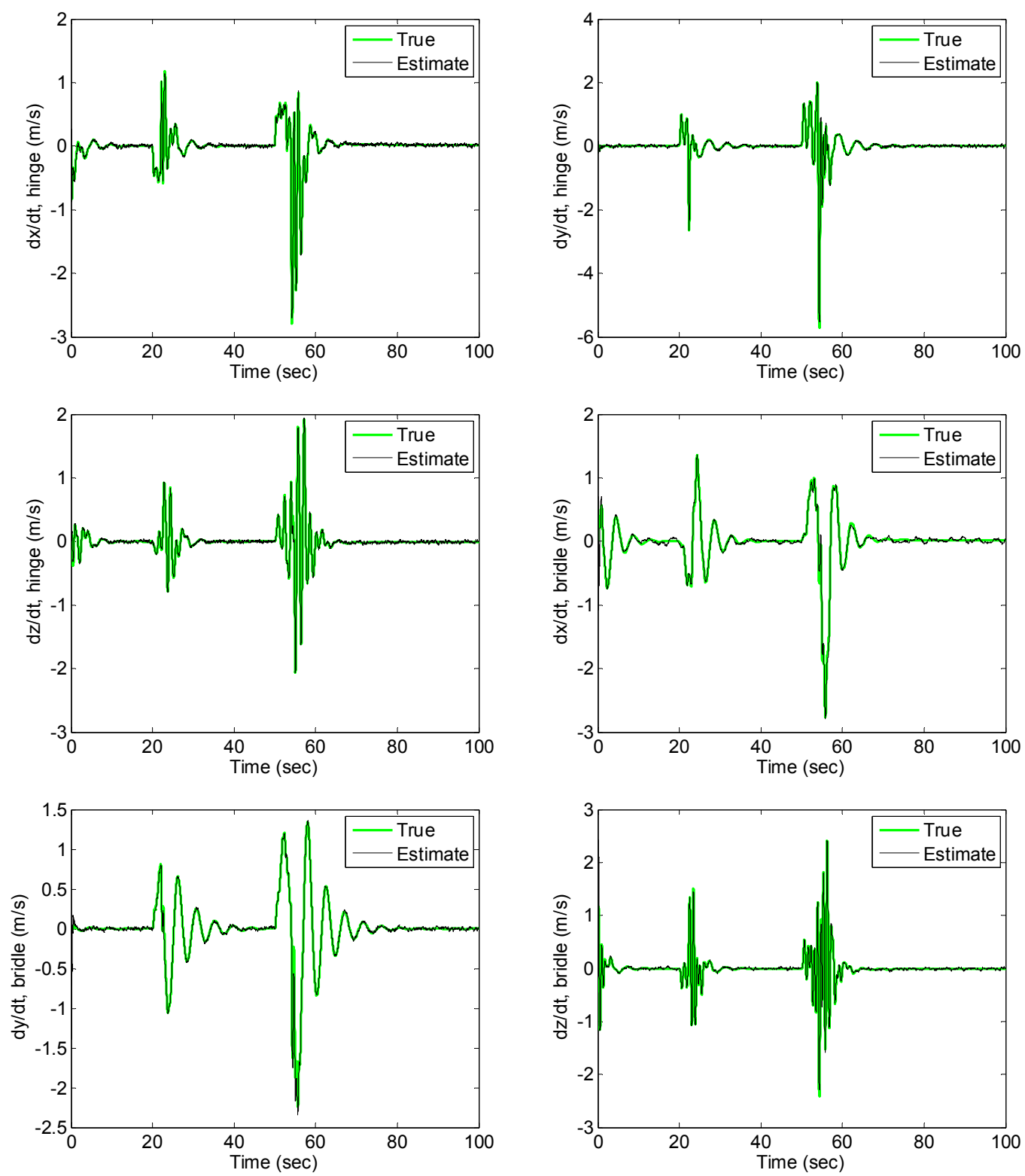

Fig. 7 Hinge point velocity time history due to attachment point disturbance, showing estimated and truth. 

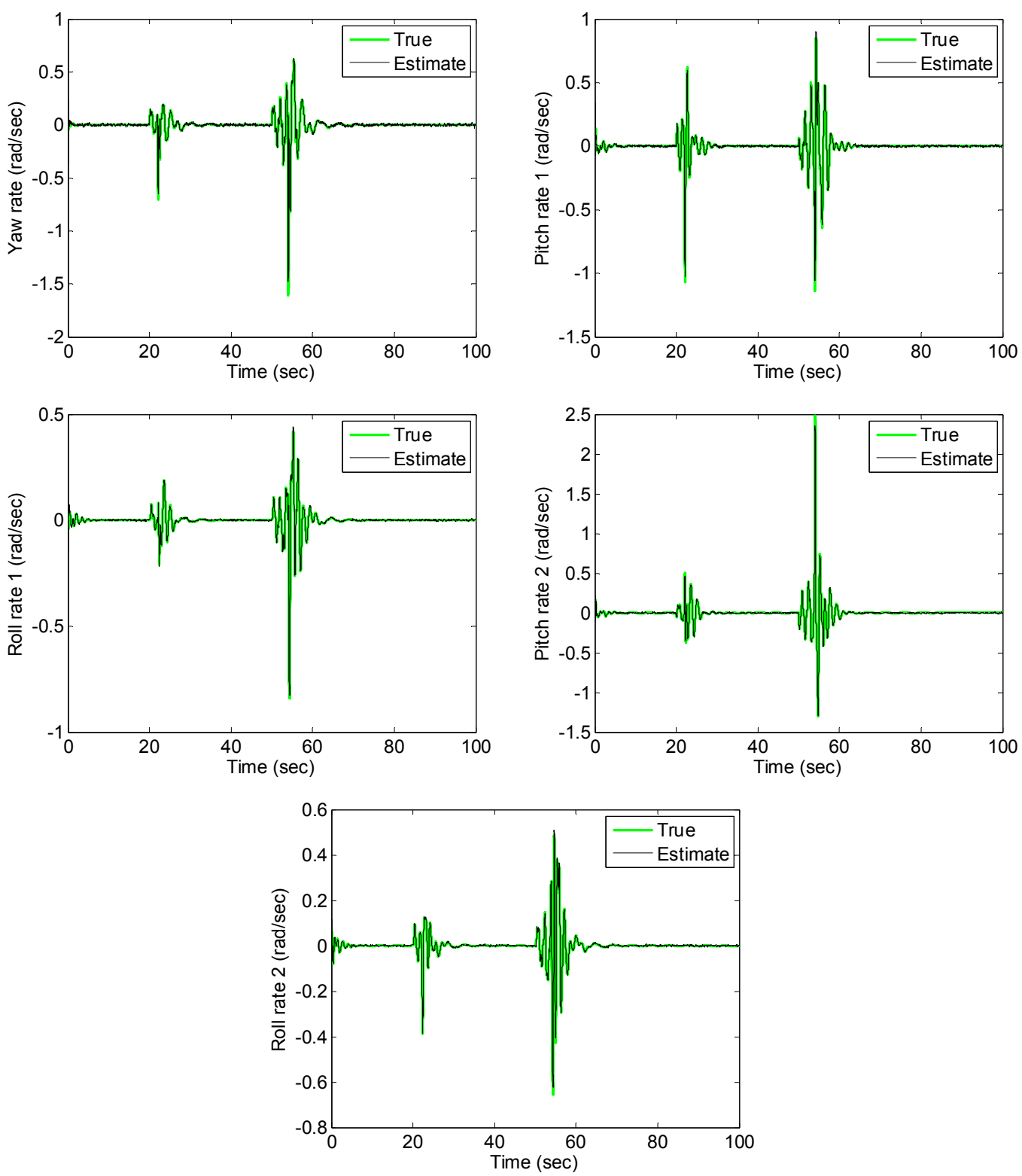

Fig. 8 Kite attitude rate time history due to attachment point disturbance, showing estimated and truth. 

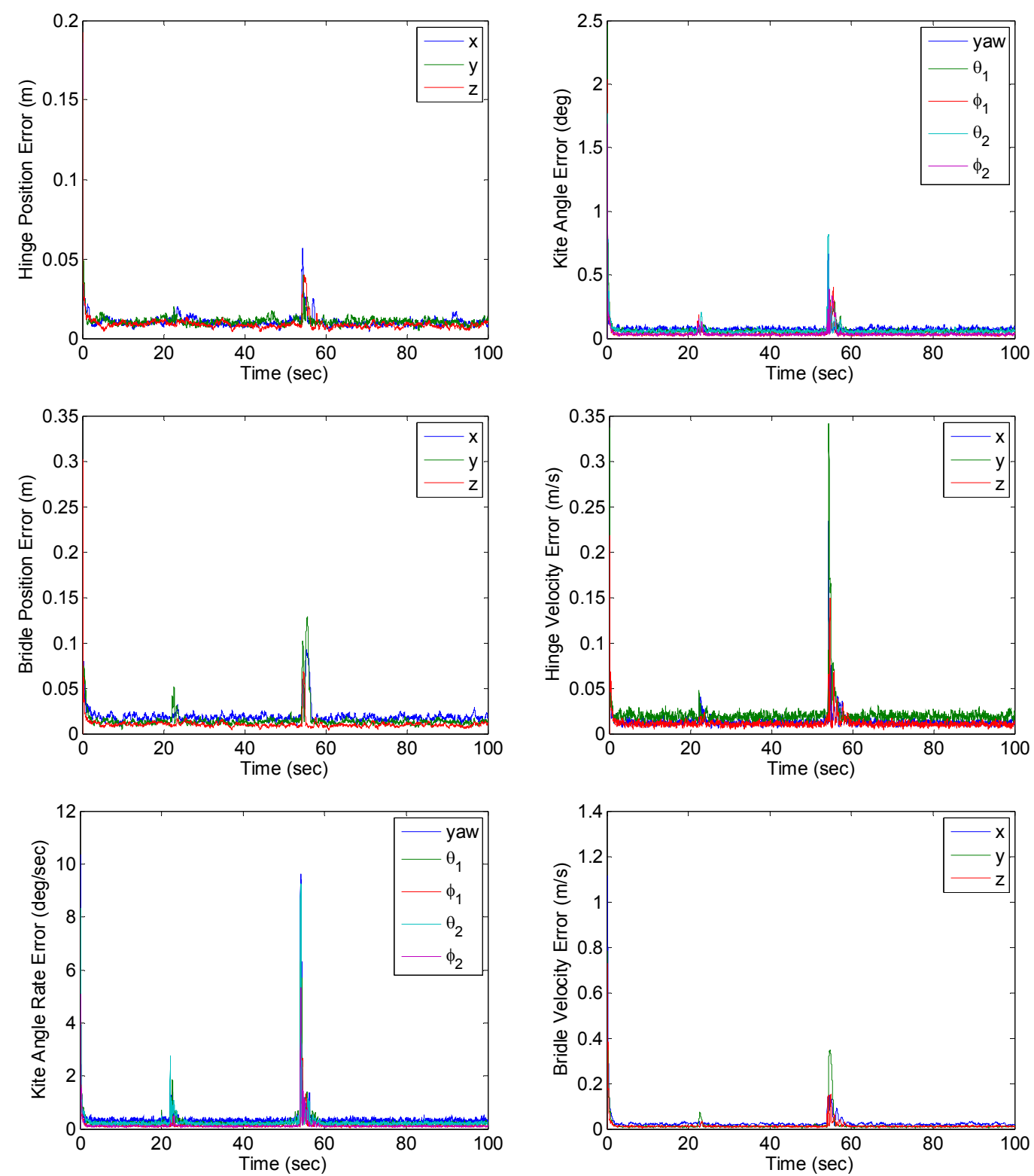

Fig. 9 Average absolute state errors for kite system obtained from 20 Monte Carlo simulations.

Fig. 9 shows the state estimation errors for the kite averaged over 20 Monte Carlo simulations with different applied measurement noise. These results show that the filter converges very rapidly from the initial state estimation errors. The errors in kite position are very small (i.e., less than $5 \mathrm{~cm}$ ) on average. The peak errors occur when the movements of the attachment points occur. The largest errors occur for the kite angular rates, where the yaw rate peaks at an error of approximately $10 \mathrm{deg} / \mathrm{sec}$.

\section{State Estimation for Cross-Wind Kite Trajectory}

In this example, the desired trajectory to be followed by the kite is given by

$$
\bar{x}_{h}=0, \quad \bar{y}_{h}=20 \sin \left(\frac{2 \pi t}{30}\right), \quad \bar{z}_{h}=0
$$

which attempts to keep the kite within single plane. The reference trajectories are derived by applying nonlinear optimal control to the kite system, as described in Part I. Numerical results for the state estimation of the kite during cross-wind kite control is shown in Fig. 10 through Fig. 13. The kite position and attitude is estimated well, with the state converging very quickly from the initial random perturbation. The kite position estimates, shown in Fig. 10, 
illustrate that the hinge point position is tracked with very little error. The kite attitude estimates are shown in Fig. 11. It can be seen that the roll angle(s) of the kite are estimated extremely well for the large cross-wind motion. The pitch of the kite is slightly more sensitive, showing smaller fluctuations in the estimate compared to the truth for both plates. The bridle position estimates are also shown in Fig. 10. Note that the position of the bridle is required to give a good estimate of the tensions in the two tethers attached to the kite. Hence, Fig. 10 shows that the bridle estimates are very good. The velocity estimates in these simulations (Fig. 12 through Fig. 13) are slightly noisier than in the previous case. In particular, the kite attitude rates show small amplitude high frequency errors superimposed on the mean. The convergence of the state estimates from the initial guess is clearly established in the case of the attitude rates, shown in Fig. 13. Fig. 14 shows the results of the absolute state errors averaged over 20 Monte Carlo simulations. These show the same level of accuracy observed in the stationkeeping case. The smoother attachment point inputs make the discrete model of the system more accurate compared to the case where the sudden attachment point movement in the previous example gives large errors in the state estimates.
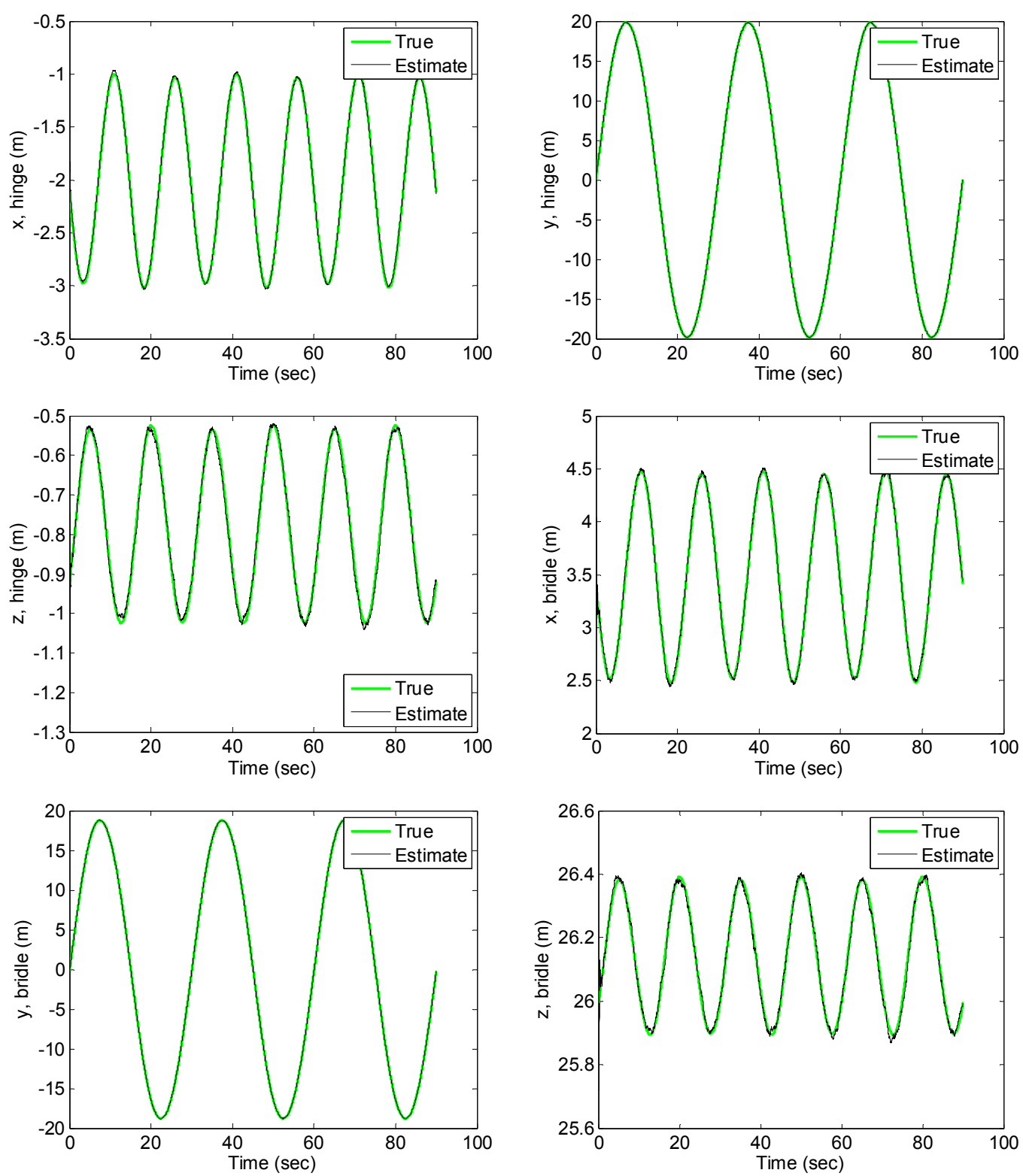

Fig. 10 Kite position time history during cross-wind motion, showing estimated and truth. 

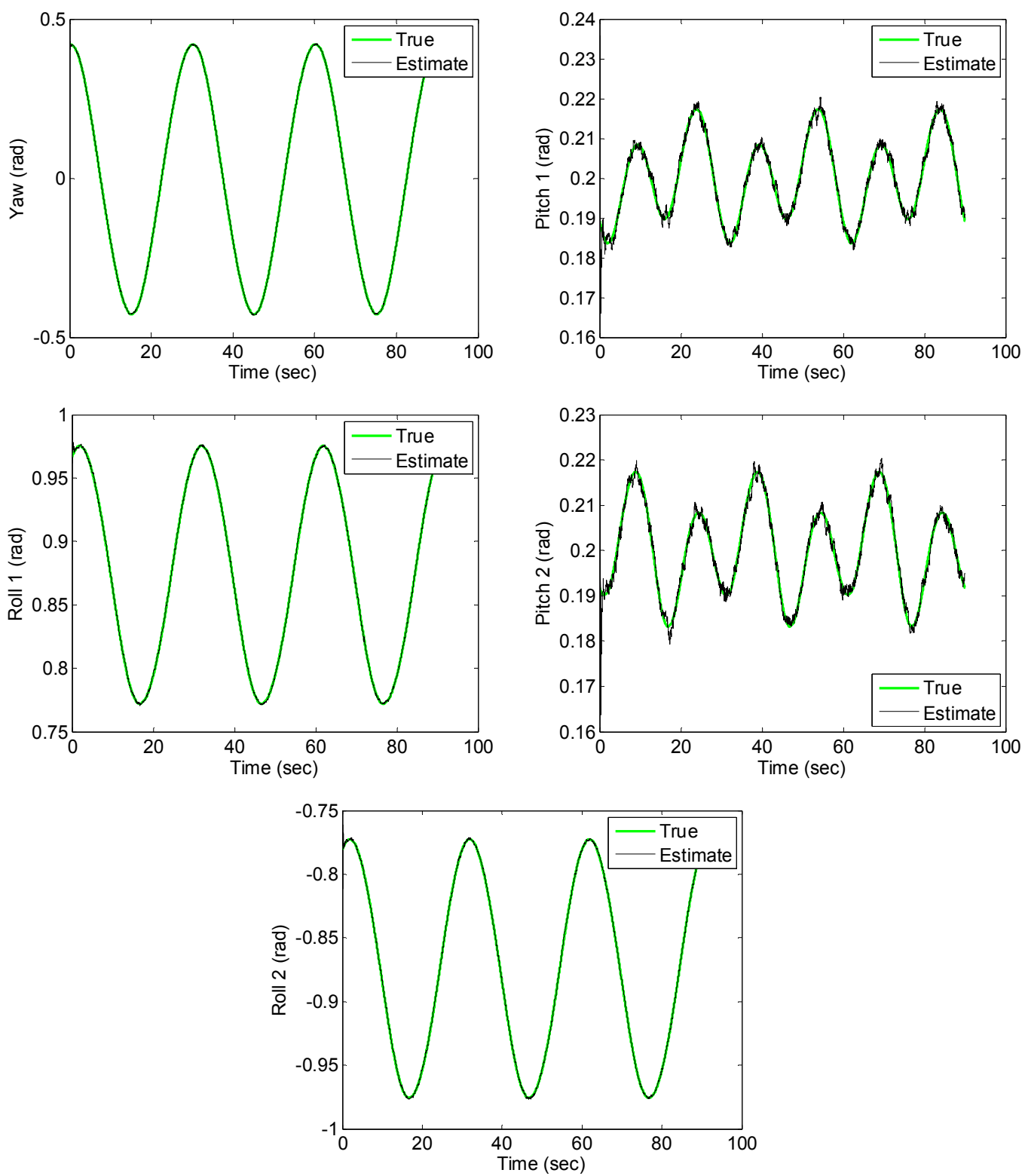

Fig. 11 Kite attitude time history during cross-wind motion, showing estimated and truth. 

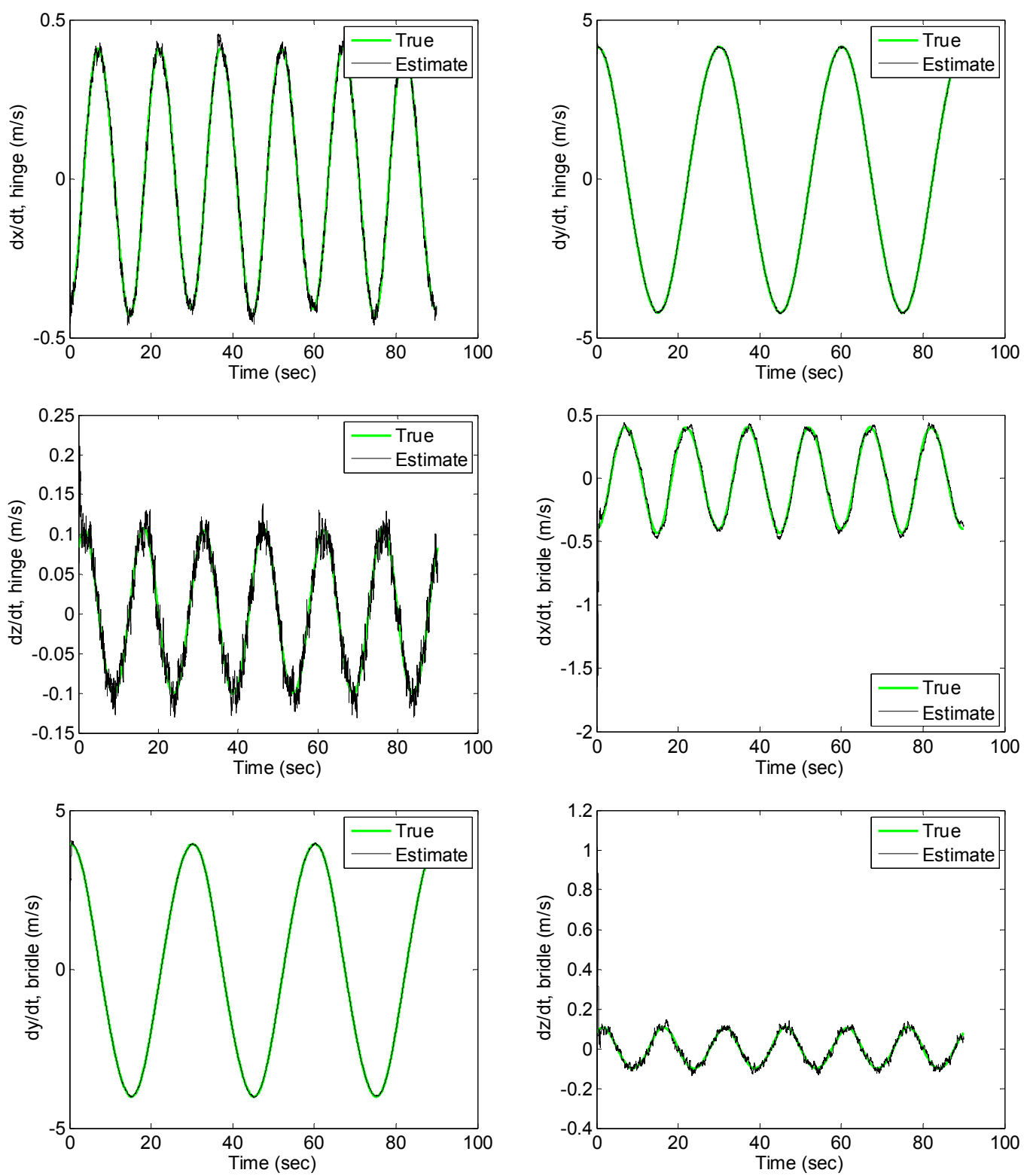

Fig. 12 Kite velocity time history during cross-wind motion, showing estimated and truth. 

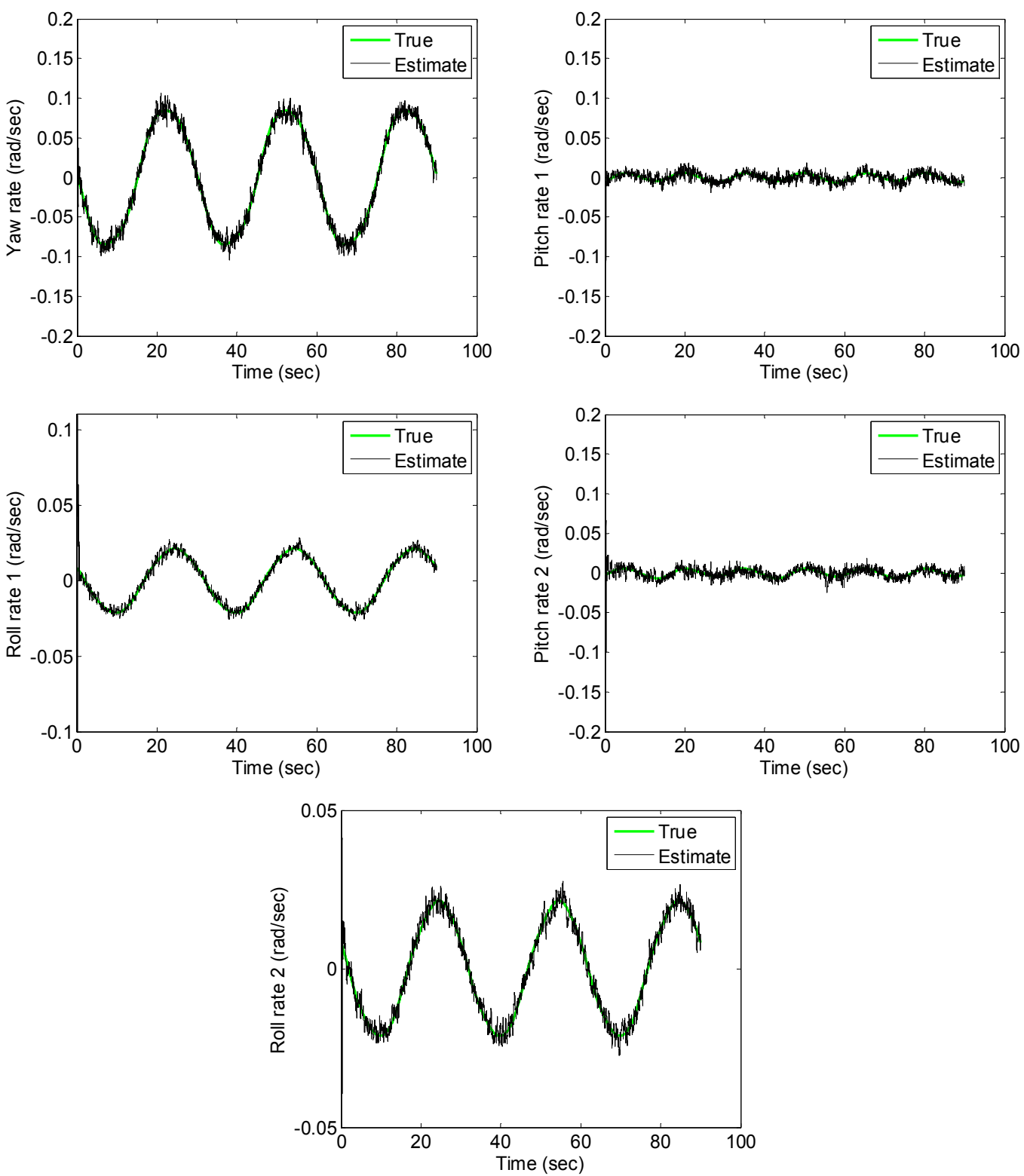

Fig. 13 Kite attitude rate time history during cross-wind motion, showing estimated and truth. 

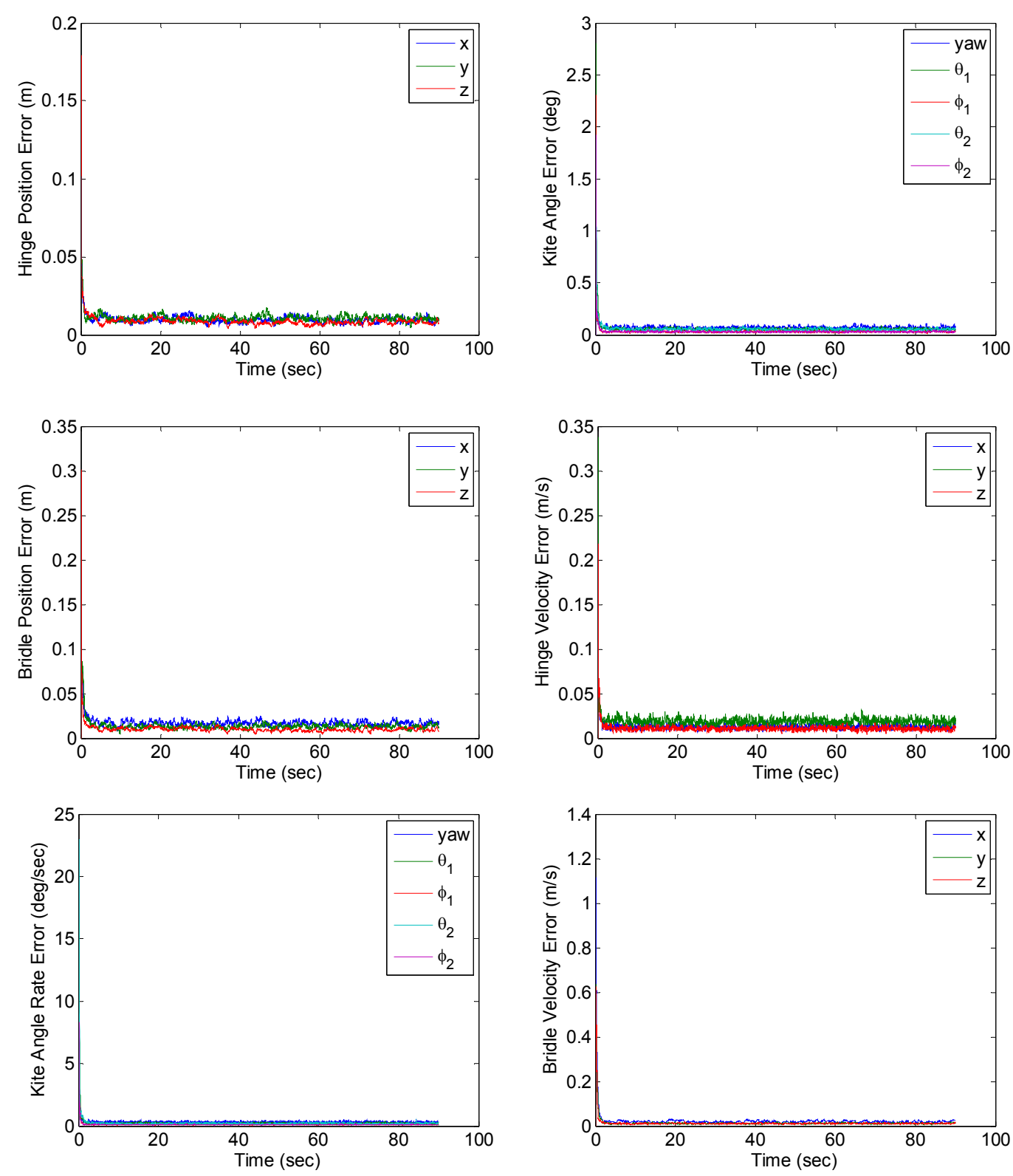

Fig. 14 Average absolute state errors for kite system during cross-wind motion obtained from 20 Monte Carlo simulations.

\section{Combined Wind Speed and Kite Estimation}

In this example, disturbances are added to the system by means of step changes in the wind speed. The stationkeeping controller is enlisted to deal with this in the true model of the system, but knowledge of the true wind speed is not used. In practice, the idea would be to use the estimated wind velocities from the kite filter in the stationkeeping controller. However, these simulations do not feed back the wind estimate. Therefore, the controller robustness is tested by applying large parameter variations from those that are assumed in the model. The goal here is to assess the estimation capability for the wind speed. The wind speed is estimated by adding an additional state representing the wind speed. The process noise assumed for the wind state is 0.5 , and the initial covariance is $10^{2}$.

The kite position estimates are shown in Fig. 15. One of the differences in these estimates compared with the previous cases is the fact that the true wind speed is not known to the filter. In fact, the initial wind estimate is -5 $\mathrm{m} / \mathrm{s}$, which is a rather sizeable error from the true value of $-10 \mathrm{~m} / \mathrm{s}$. The $x$ - and $z$-positions of the kite are estimated extremely well. The cross-wind position estimate is also very good. However, because there is virtually no cross- 
wind motion in the results, the noise in the estimate stands out on the scale shown. This can be interpreted as the estimation error. The kite attitude estimates are shown in Fig. 16, which also shows that the kite is stabilized quite well in response to the step changes in wind speed. It is interesting to note that the kite rolls slightly in response to the wind speed changes. This is due to the fact that the kite is controlled by movement of the attachment points. Any small differences in the attachment point positions on each side of the kite create a rolling moment on the kite. However, the variations in roll angle are relatively small and are accurately predicted in the state estimates. Fig. 16 shows that the pitch of the kite reaches equilibrium very quickly after the wind speed changes, again illustrating the effectiveness of the controller. The bridle position estimates are shown in Fig. 15, which again show that the estimation is very good. The rate estimates are shown in Fig. 17 through Fig. 18. These results show some larger errors at the instants where the wind changes speed. This is understandable because the wind estimate does not change instantaneously, as shown in Fig. 18. The initial convergence of the wind estimate is extremely good, and the step changes in the wind speed are captured excellently in the estimates. The results clearly show the filter is able to detect changes in parameters quite quickly with little adverse effect on the resulting estimate of the kite state vector.
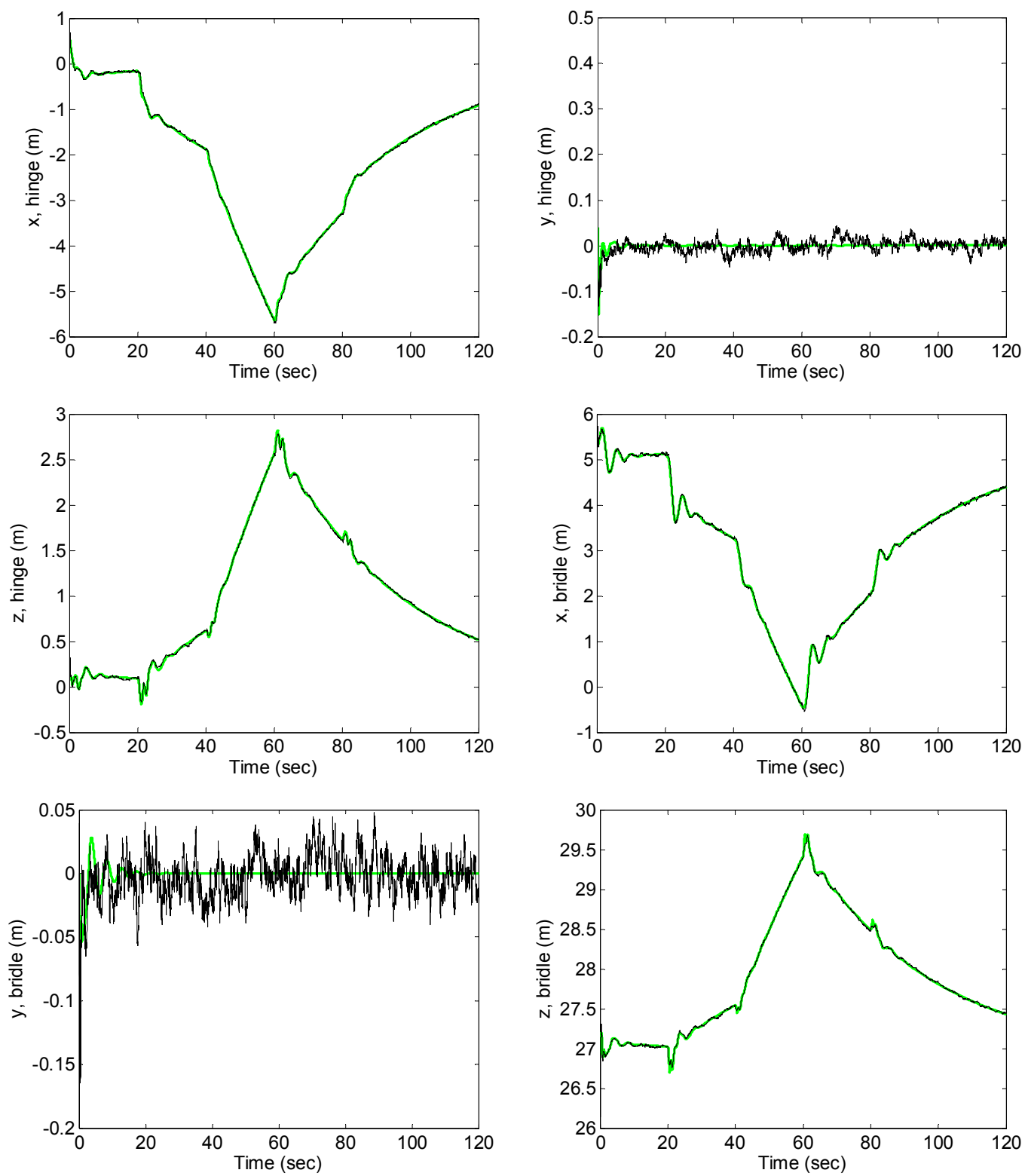

Fig. 15 Kite position time history during with changes in wind speed, showing estimated and truth. 

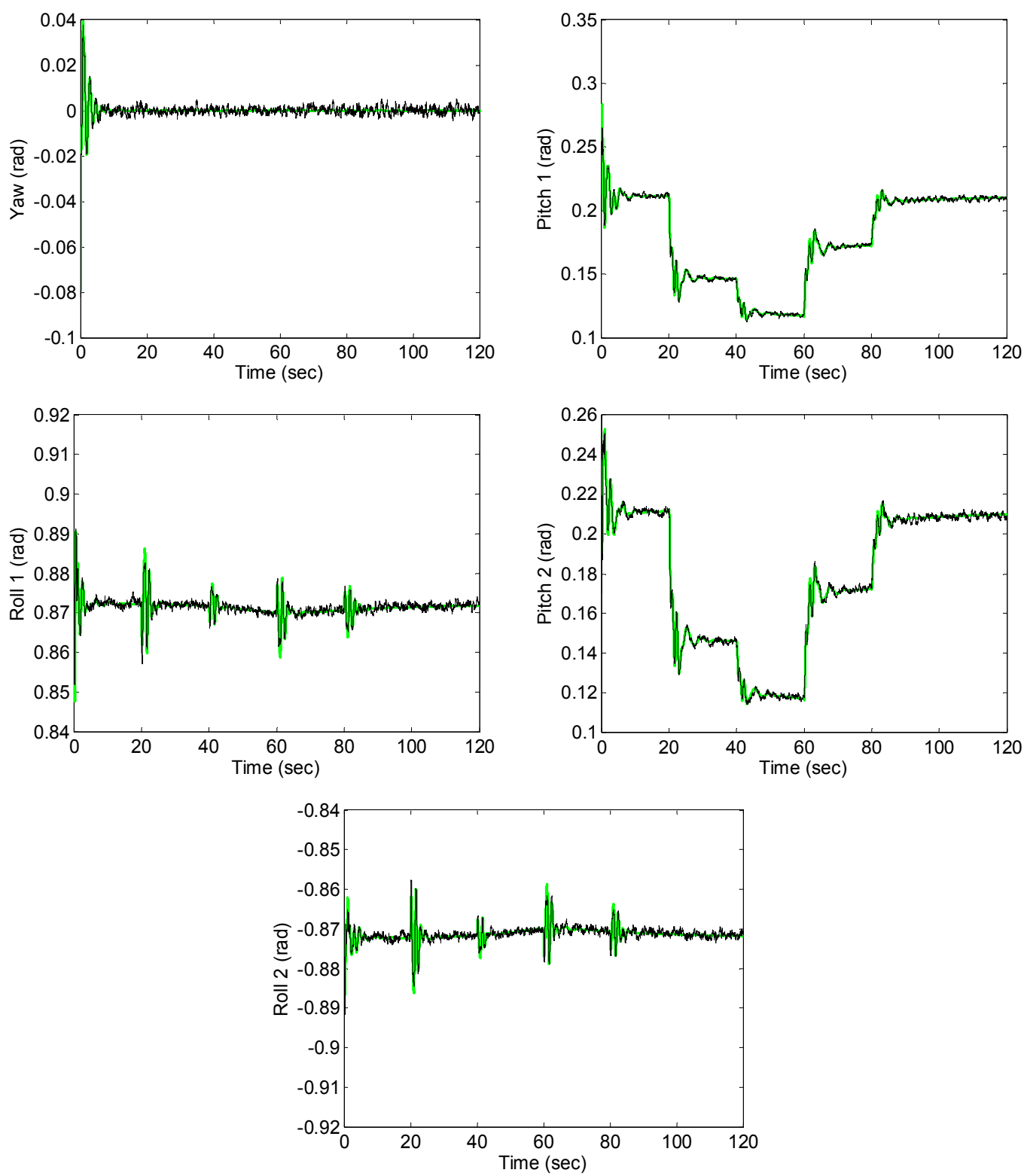

Fig. 16 Kite attitude time history during with changes in wind speed, showing estimated and truth. 

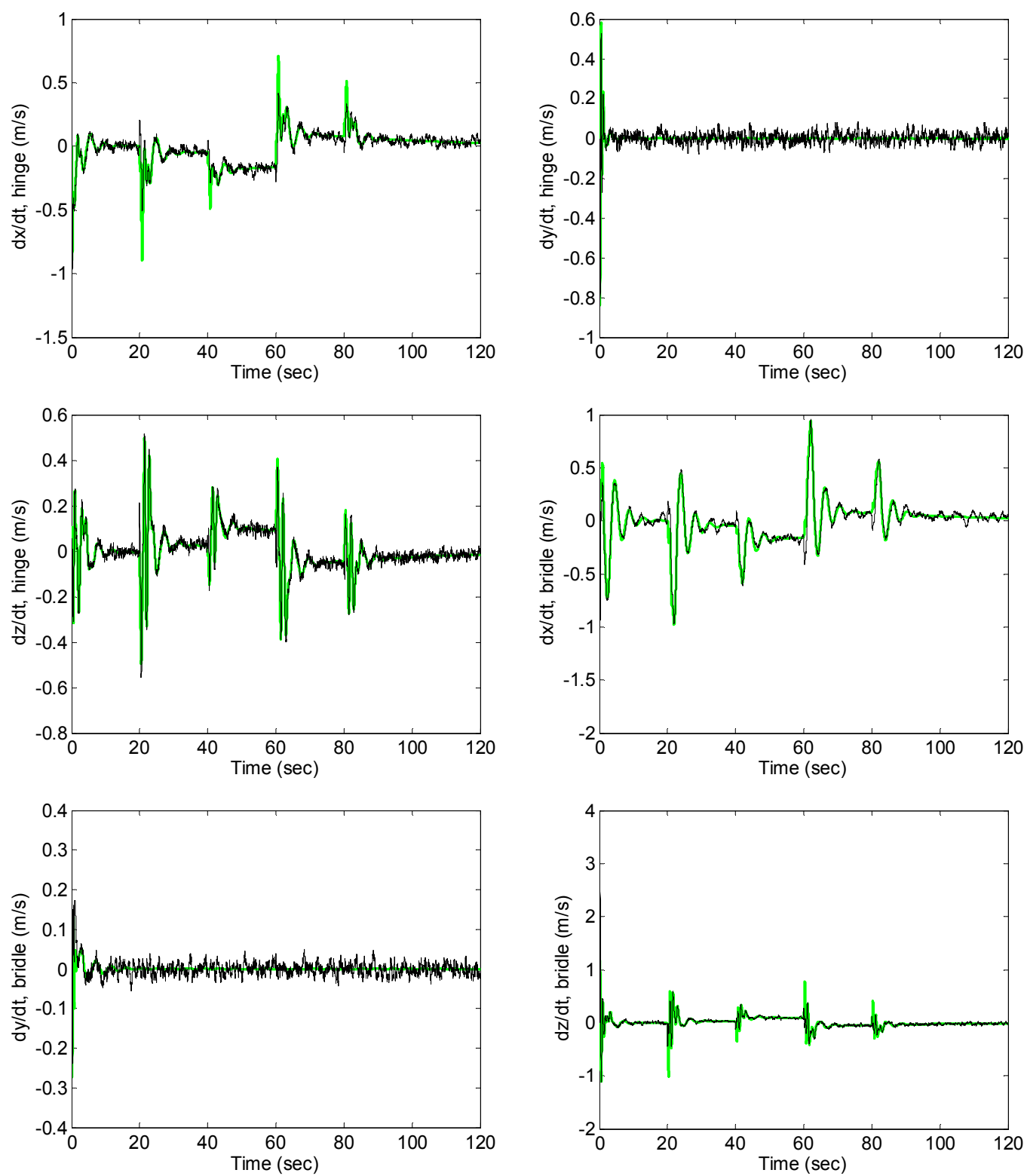

Fig. 17 Kite velocity time history during with changes in wind speed, showing estimated and truth. 

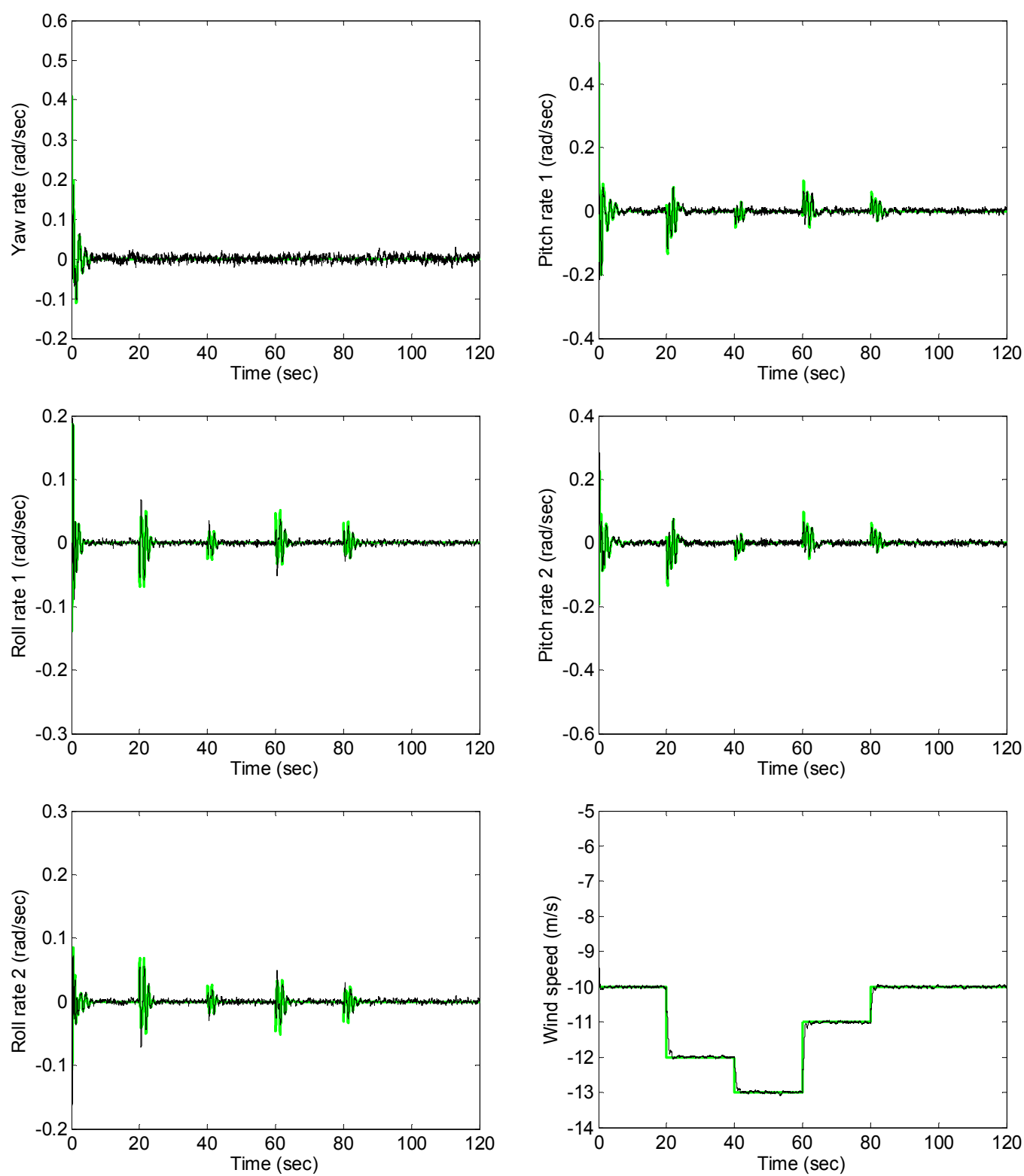

Fig. 18 Kite attitude rate time history during simulation with changes in wind speed, showing estimated and truth. 

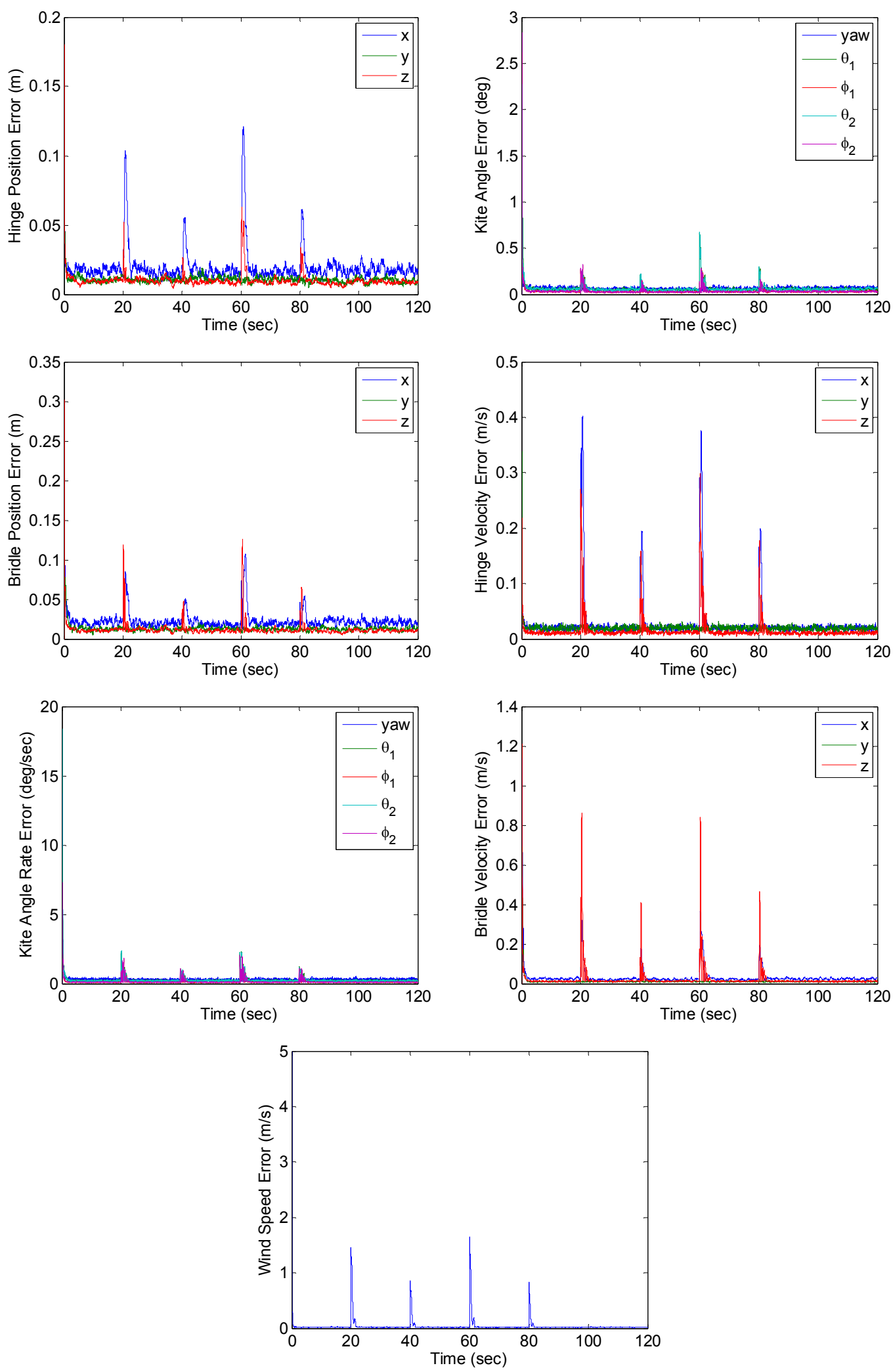

Fig. 19 Average absolute state errors for kite system during simulation with changes in wind speed obtained from 20 Monte Carlo simulations 
Fig. 19 shows the average absolute state estimation errors from 20 Monte Carlo simulations. The mean state errors are very similar to the previous results, except from the instants where the wind speed changes. The error in the wind speed estimate peaks to an amount that is slightly less than the absolute change in the wind speed. This is caused by the lag of the filter due to its inability to predict changes in states for which there is no direct measurement or control input. However, the error decreases to the mean of about $0.02 \mathrm{~m} / \mathrm{s}$ after a period of $2 \mathrm{sec}$, on average. These results clearly illustrate the ability to estimate both the full kite state vector as well as the wind speed with only a subset of measurements. The filter may prove useful for estimating some properties of real kite systems from experimental measurements.

\section{Conclusions}

Dynamic state estimation for a tethered kite has been demonstrated using a simplified kite model. The estimation has been carried out in a simulation environment with control over the system parameters. The kite model treats the kite as composed of two flat plates that are controlled by moving the position of the tether attachment points. A square-root version of the unscented Kalman filter is used to provide full state estimates given a limited, noisy observation vector. The filter provides a combination of good stability and accuracy without the need to derive analytic Jacobians. This is extremely important for the kite model, which is highly nonlinear. The filter is also able to act as a nonlinear observer for the wind speed, which is accurately estimated.

\section{References}

${ }^{1}$ Ockels, W.J., "Laddermill, a Novel Concept to Exploit the Energy in the Airspace," Aircraft Design, Vol. 4, 2001, pp.81-97.

${ }^{2}$ Meijaard, J.P., Ockels, W.J., and Schwab, A.L., "Modelling of the Dynamic Behaviour of a Laddermill, A Novel Concept to Exploit Wind Energy," Proceedings of the Third International Symposium on Cable Dynamics, Norway, Aug. 1999, pp.229-234.

${ }^{3}$ Lansdorp, B., and Ockels, W.J., "Comparison of Concepts for High-Altitude Wind Energy Generation with Ground Based Generator," Proceedings of the NRE 2005 Conference, Beijing, China, pp.409-417.

${ }^{4}$ Lansdorp, B., Remes, B., and Ockels, W.J., "Design and Testing of a Remotely Controlled Surfkite for the Laddermill,” World Wind Energy Conference, Melbourne, Australia, Nov. 2005.

${ }^{5}$ Lansdorp, B., and Williams, P., "The Laddermill - Innovative Wind Energy from High Altitudes in Holland and Australia," Paper presented at Wind Power 2006, Adelaide, Australia, September 2006.

${ }^{6}$ Carpenter, H.G., "Tethered Aircraft Having Remotely Controlled Angle of Attack," US Patent 5,931,416.

${ }^{7}$ Carpenter, H.G., "Tethered Aircraft System for Gathering Energy From Wind," US Patent 6,254,034.

${ }^{8}$ Roberts, B.W., and Shepard, D.H., "Unmanned Rotorcraft to Generate Electricity using Upper Atmospheric Winds," Australian International Aerospace Congress, Brisbane, July 2003.

${ }^{9}$ Etkin, B., Dynamics of Atmospheric Flight, Wiley, New York, 1972.

${ }^{10}$ Riegler, G., and Riedler, W., "Tethered Wind Systems for the Generation of Electricity," Journal of Solar Energy Engineering, Vol. 106, 1984, pp.177-181.

${ }^{11}$ Riegler, G., Riedler, W., and Horvath, E., "Transformation of Wind Energy by a High-Altitude Power Plant," Journal of Energy, Vol. 7, No. 1, 1983, pp.92-94.

${ }^{12}$ Fletcher, C.A.J., and Roberts, B.W., "Electricity Generation from Jet-Stream Winds," Journal of Energy, Vol. 3, 1979, pp.241-249.

${ }^{13}$ Fletcher, C.A.J., "On the Rotary Wing Concept for Jet Stream Electricity Generation," Journal of Energy, Vol. 7, No. 1, 1983, pp.90-92.

${ }^{14}$ Rye, D.C., "Longitudinal Stability of a Hovering, Tethered Rotorcraft," Journal of Guidance, Control, and Dynamics, Vol. 8, No. 6, 1985, pp.743-752.

${ }^{15}$ Fry, C.M., and Hise, H.W., "Wind Driven, High Altitude Power Apparatus," US Patent 4,084,102, April 1978.

${ }^{16}$ Kling, A., "Wind Driven Power Plant," US Patent 4,073,516, Feb. 1978.

${ }^{17}$ Pugh, P.F., "Wind Generator Kite System," US Patent 4,486,669, Dec. 1984.

${ }^{18}$ Biscomb, L.I., "Multiple Wind Turbine Tethered Airfoil Wind Energy Conversion System," US Patent 4,285,481, Aug. 1981.

${ }^{19}$ Watson, W.K., "Airship-Floated Wind Turbine," US Patent 4,491,739, Jan. 1985.

${ }^{20}$ Shepard, D.H., "Power Generation from High Altitude Winds," US Patent 4,659,940, April 1987.

${ }^{21}$ Rundle, C.V., "Tethered Rotary Kite," US Patent 5,149,020, Sept. 1992.

${ }^{22}$ Roberts, B.W., "Windmill Kite," US Patent 6,781,254, Aug. 2004.

${ }^{23}$ Mouton, W.J., and Thompson, D.F., “Airship Power Turbine,” US Patent 4,166,596, Sept. 1979. 
${ }^{24}$ Bolonkin, A., "Utilization of Wind Energy at High Altitude,” AIAA Paper 2004-5705, Aug. 2004.

${ }^{25}$ Loyd, M.L., "Crosswind Kite Power," Journal of Energy, Vol. 4, No. 3, 1980, pp.106-111.

${ }^{26}$ Payne, P.R., and McCutchen, C., "Self-Erecting Windmill," US Patent 3,987,987, Oct. 1976.

${ }^{27}$ Loeb, A., "Wind Driven Energy System," US Patent 4,124,182, Nov. 1978.

${ }^{28}$ Lois, L., "Apparatus for Extracting Energy from Winds at Significant Height Above the Surface," US Patent 4,076,190, Feb. 1978.

${ }^{29}$ Williams, P., Lansdorp, B., and Ockels, W., "Optimal Cross-Wind Towing and Power Generation with Tethered Kites," submitted to AIAA Guidance, Navigation and Control Conference, Aug. 2007.

${ }^{30}$ Williams, P., Lansdorp, B., and Ockels, W., "Flexible Tethered Kite with Moveable Attachment Points I: Dynamics and Control," submitted to AIAA Atmospheric Flight Mechanics Conference, Aug. 2007.

${ }^{31}$ Kalman, R.E., "A New Approach to Linear Filtering and Prediction Problems," Transactions of the ASME, Ser. D, Journal of Basic Engineering, Vol. 82, 1960, pp.35-45.

${ }^{32}$ Handschin, J.E., and Mayne, D.Q., "Monte Carlo Techniques to Estimate the Conditional Expectation in MultiStage Non-Linear Filtering," International Journal of Control, Vol. 9, 1969, pp.547-559.

${ }^{33}$ Julier, S.J., Uhlmann, J.K., and Durrant-Whyte, H., "A New Approach for Filtering Nonlinear Systems," in Proceedings of the American Control Conference, 1995, pp. 1628-1632.

${ }^{34}$ Kaminski, P.G., Bryson, A.E., and Schmidt, S.F., "Discrete Square Root Filtering: A Survey of Current Techniques,"' IEEE Transactions on Automatic Control, Vol. 16, 1971, pp.727-736.

${ }^{35}$ van der Merwe, R. and Wan, E.A., "The Square-Root Unscented Kalman Filter for State and ParameterEstimation," in Proceedings of International Conference on Acoustics, Speech, and Signal Processing, Salt Lake City, UT, May 2001. 